\title{
Propriedades de controladores fuzzy: um estudo de caso
}

\author{
Properties of fuzzy controllers: a case study \\ V. L. D. Mattos ${ }^{1}$; L. C. Coelho ${ }^{2}$ \\ ${ }^{I}$ Instituto de Matemática, Estatística e Física, FURG, CEP: 96203-900, Rio Grande-RS, Brasil \\ ${ }^{2}$ Escola de Engenharia, FURG, CEP: 96203-900, Rio Grande-RS, Brasil \\ vivianemattos@furg.br
}

(Recebido em 14 de setembro de 2014; aceito em 29 de dezembro de 2014)

\begin{abstract}
Este estudo foi desenvolvido para avaliar a influência da utilização de diferentes funções de pertinência e diferentes métodos de "defuzzificação" na mensuração de um construto por meio de controladores fuzzy, os quais são especialmente indicados para mensurar variáveis complexas ou mal definidas. Foi utilizado um experimento planejado, como um projeto fatorial completo do tipo $2 \times 3$, que considerou as funções de pertinência triangular e gaussiana para "fuzzificação", além dos métodos do centróide, dos máximos e da média dos máximos para "defuzzificação". O estudo considerou 14 questionários, extraídos aleatoriamente na amostra de validação do modelo, que foram analisados nas seis condições experimentais. Não foram encontradas evidências da influência dos fatores sobre os resultados. Uma análise complementar com o coeficiente de Cronbach identifica o método da média dos máximos e a função de pertinência triangular como geradores de resultados mais fidedignos.

Palavras-chave: qualidade, satisfação, lógica fuzzy, projeto de experimentos.
\end{abstract}

This study was done to evaluate the influence of using different membership functions and different defuzzification methods in the measurement of a construct by fuzzy controllers. They are particularly suitable for measuring complex or ill-defined variables. An $2 \times 3$ full factorial experiment consider the triangular membership functions and the gaussian membership functions for fuzzification and the centroid method, the maximum method and the mean of maximum method for defuzzification. The study used 14 instruments, randomly selected at the validation sample of the model, which were analyzed in the six experimental conditions. They weren't found evidence of the influence of these factors on the results. A complementary analysis with Cronbach coefficient identifies the mean of maximum method and the triangular membership function to obtain more reliable results.

Keywords: quality, satisfaction, fuzzy logic, experimental design.

\section{INTRODUÇÃO}

O desenvolvimento de uma análise de dados fidedigna é de fundamental importância para a maioria das organizações por fornecer subsídios para a tomada de decisão. Entretanto, seu resultado pode ser bastante influenciado pela precisão da mensuração das variáveis envolvidas. Muitas vezes, esta precisão é difícil de ser obtida, principalmente se as variáveis forem complexas ou mal definidas. Segundo Zadeh [1], de acordo com o princípio da incompatibilidade, alta complexidade é incompatível com alta precisão.

Neste contexto, a lógica fuzzy assume importante papel por fornecer meios para lidar com a ideia do nebuloso, do vago. De acordo com Malutta [2], por meio dessa teoria uma mensuração pode ser feita de maneira mais coerente do que na lógica clássica, pois existem diferentes graus de verdade, variando entre o puramente verdadeiro e o puramente falso, o que reduz a perda de informações e, em muitos casos, reflete melhor a realidade. Uma técnica que tem sido bastante utilizada na mensuração desse tipo de variável é a dos controladores fuzzy, desenvolvidos em três estágios: "fuzzificação" das entradas discretas, processamento através da inferência fuzzy e cálculo de saídas discretas por "defuzzificação".

Vários trabalhos já foram publicados relatando aplicações de controladores fuzzy com sucesso. Em Simões et al. [3] eles são utilizados no desenvolvimento de um sistema para geração de energia eólica capturada por uma turbina de eixo vertical; em Souto [4], para a 
definição de faixas de desempenho para indicadores em um sistema especialista que gerencia o funcionamento de uma usina nuclear; em Abadi e Khooban [5], para rastreamento de trajetória de robôs móveis e em Sanaye et al. [6], para estudar o conforto térmico em uma cabine de um automóvel.

Na utilização desses controladores, o processo de "fuzzificação" tem a função de transformar entradas discretas em saídas nebulosas, com diferentes graus de pertinência aos conjuntos fuzzy, os quais representam termos linguísticos. Para fazer essa transformação é necessário que sejam definidos os termos linguísticos a serem considerados na mensuração da variável, bem como a respectiva função de pertinência, que os definem como conjuntos fuzzy. No processo de "defuzzificação", o resultado fuzzy é transformado em uma saída discreta, o que pode ser feito de diversas maneiras.

O presente estudo analisa a influência da utilização de duas funções de pertinência (função gaussiana e função triangular) e três métodos de "defuzzificação" (método dos máximos, método da média dos máximos e método do centróide) em um modelo conceitual para a satisfação do consumidor, que utiliza controladores fuzzy.

\section{MATERIAL E MÉTODOS}

Este estudo está inserido em um processo de construção de um modelo conceitual, fundamentado na lógica fuzzy, para mensurar, por meio de controladores fuzzy, a satisfação dos consumidores na utilização de um serviço. Mais detalhes podem ser encontrados em Mattos et al. [7] e Viticoski et al. [8]. No modelo considerado, o nível de satisfação do consumidor é determinado por meio da expressão (1):

$$
\text { nível de satisfação }=0,70 f_{1}+0,12 f_{2}+0,10 f_{3}+0,08 f_{4} \text {, }
$$

onde: $f_{1}$ representa o fator atendimento, $f_{2}$ representa o fator local de embarque/desembarque, $f_{3}$ representa o fator veículo e $f_{4}$ representa o fator segurança. Os pesos foram definidos por especialistas levando em consideração as percentagens explicadas da variância por cada fator, encontradas na análise fatorial desenvolvida na validação de construto do modelo. Já a pontuação atribuída a cada fator é determinada por controladores fuzzy a partir da avaliação de vários itens.

Nesses controladores, o universo de discurso é definido por $U=[0 ; 100]$, o conjunto de termos linguísticos para avaliação do nível de satisfação é definido por $T\left(x_{1}\right)=\{$ muito insatisfeito; insatisfeito; indiferente; satisfeito; muito satisfeito $\}, \mathrm{e}$ o conjunto considerado na mensuração da importância do fator por $T\left(x_{2}\right)=\{$ muito pequena; pequena; média, grande $\}$. As regras de inferência e suas respectivas forças são definidas por especialistas, enquanto que o método de inferência utilizado é o de Mamdani, o mais citado na literatura.

A função de pertinência e o método de "defuzzificação" são definidos pela análise dos resultados de um experimento planejado na forma de projeto fatorial completo do tipo $2 \times 3$. $\mathrm{O}$ fator função de pertinência é considerado em dois níveis: função triangular e função gaussiana, enquanto que para o método de "defuzzificação" são utilizados três níveis: método do centróide, método dos máximos e método da média dos máximos. Quatorze questionários são extraídos aleatoriamente da amostra utilizada na validação de construto do modelo e as informações são analisadas em cada uma das seis condições experimentais. Os conjuntos fuzzy que representam os termos linguísticos considerados nessa análise estão apresentados nos quadros 1 e 2 .

Os métodos de "defuzzificação" considerados nesse estudo estão apresentados a seguir.

O Método dos Máximos transforma a saída fuzzy em saída discreta identificando o core do conjunto fuzzy ao qual o elemento pertence com maior grau de pertinência. Se o valor máximo não for único, considera-se o ponto central do intervalo de cores. Considera-se que o core de um 
conjunto fuzzy $\mathrm{N}$ consiste em todos os elementos que pertencem ao universo de discurso cujo grau de pertinência é igual a 1, ou seja: $\operatorname{Core}(N)=\left\{y \in I \mid \mu_{N}(y)=1\right\}$.

Quadro 1 - Conjuntos fuzzy dos termos linguísticos que representam o nível de satisfação

\begin{tabular}{|c|c|c|c|c|c|}
\hline TL & \multicolumn{3}{|c|}{ Função triangular } & \multicolumn{2}{|r|}{ Função gaussiana } \\
\hline $\begin{array}{l}\text { Muito } \\
\text { Insatisfeito }\end{array}$ & $\mu_{T L}(x)=$ & $\left\{\begin{array}{l}0, \\
1, \\
\frac{30-x}{20} \\
0,\end{array}\right.$ & $\begin{array}{ll}\text { se } & x<0 \\
\text { se } & 0 \leq x<10 \\
\text {, se } & 10 \leq x \leq 30 \\
\text { se } & x>30\end{array}$ & $\mu_{T L}(x)=$ & $\begin{cases}0, & \text { se } x<0 \\
1, & \text { se } 0 \leq x<10 \\
e^{-\frac{1}{2\left(\frac{x-10}{6,67}\right)^{2}}}, & \text { se } 10 \leq x<30 \\
0, & \text { se } x \geq 30\end{cases}$ \\
\hline Insatisfeito & $\mu_{T L}(x)=$ & $\left\{\begin{array}{l}0, \\
\frac{x-10}{20} \\
\frac{50-x}{20} \\
0,\end{array}\right.$ & $\begin{array}{ll}\text { se } & x<10 \\
\text { se } & 10 \leq x<30 \\
\text { se } & x=30 \\
\text { se } & 30<x \leq 50 \\
\text { se } x>50\end{array}$ & $\mu_{T L}(x)=$ & $\begin{cases}0, & \text { se } x<10 \\
e^{-\frac{1}{2}\left(\frac{x-30}{6,67}\right)^{2}} & \text { se } 10 \leq x<50 \\
0, & \text { se } x \geq 50\end{cases}$ \\
\hline Indiferente & $\mu_{T L}(x)=$ & $\left\{\begin{array}{l}0, \\
\frac{x-30}{20} \\
\frac{70-x}{20} \\
0\end{array}\right.$ & $\begin{array}{ll}\text { se } & x<30 \\
\text { se } & 30 \leq x<50 \\
\text { se } & x=50 \\
\text { se } & 50<x \leq 70 \\
\text { se } & x>70\end{array}$ & $\mu_{T L}(x)=$ & $\begin{cases}0, & \text { se } x<30 \\
e^{-\frac{1}{2}\left(\frac{x-50}{6,67}\right)^{2}} & \text { se } 30 \leq x<70 \\
0, & \text { se } x \geq 70\end{cases}$ \\
\hline Satisfeito & $\mu_{T L}(x)=$ & $\left\{\begin{array}{l}0, \\
\frac{x-50}{20} \\
\frac{90-x}{20} \\
0\end{array}\right.$ & $\begin{array}{l}\text { se } x<50 \\
\text { se } 50 \leq x<70 \\
\text { se } x=70 \\
\text { se } 70<x \leq 90 \\
\text { se } x>90\end{array}$ & $\mu_{T L}(x)=$ & $\begin{cases}0, & \text { se } x<50 \\
e^{-\frac{1}{2}\left(\frac{x-70}{6,67}\right)^{2}} & \text { se } 50 \leq x<90 \\
0, & \text { se } x \geq 90\end{cases}$ \\
\hline $\begin{array}{l}\text { Muito } \\
\text { Satisfeito }\end{array}$ & $\mu_{T L}(x)=$ & $\left\{\begin{array}{l}0 \\
\frac{x-70}{20} \\
1 \\
0\end{array}\right.$ & $\begin{array}{ll}\text { se } & x<70 \\
\text { se } & 70 \leq x<90 \\
\text { se } & 90 \leq x \leq 100 \\
\text { se } & x>100\end{array}$ & $\mu_{T L}(x)=$ & $\begin{cases}0, & \text { se } x<70 \\
e^{-\frac{1}{2}\left(\frac{x-90}{6,67}\right)^{2}} & \text { se } 0 \leq x<90 \\
1, & \text { se } 90 \leq x \leq 100 \\
0, & \text { se } x>100\end{cases}$ \\
\hline
\end{tabular}

OBS: TL significa termo linguístico. 
Quadro 2 - Conjuntos fuzzy dos termos linguísticos que representam a importância do fator

\begin{tabular}{|c|c|c|c|c|}
\hline TL & \multicolumn{2}{|r|}{ Função triangular } & \multicolumn{2}{|r|}{ Função gaussiana } \\
\hline $\begin{array}{l}\text { Muito } \\
\text { Pequena }\end{array}$ & $\mu_{T L}(x)=$ & $\left\{\begin{array}{lll}0, & \text { se } x<0 \\
1, & \text { se } & 0 \leq x<5 \\
\frac{20-x}{15} & \text { se } & 5 \leq x \leq 20 \\
0, & \text { se } & x>20\end{array}\right.$ & $\mu_{T L}(x)=$ & $\left\{\begin{array}{lll}0, & \text { se } x<0 \\
1, & \text { se } 0 \leq x<5 \\
e^{-\frac{1}{2}\left(\frac{x-5}{5}\right)^{2}} & \text { se } 5 \leq x<20 \\
0, & \text { se } x \geq 20\end{array}\right.$ \\
\hline Pequena & $\mu_{T L}(x)=$ & $\left\{\begin{array}{l}0, \quad \text { se } x<5 \\
\frac{x-5}{15}, \text { se } 5 \leq x<20 \\
1, \quad \text { se } x=20 \\
\frac{50-x}{30}, \text { se } \quad 20<x \leq 50 \\
0, \quad \text { se } x>50\end{array}\right.$ & $\mu_{T L}(x)=$ & $\left\{\begin{array}{lll}0, & \text { se } x<5 \\
e^{-\frac{1}{2}\left(\frac{x-20}{5}\right)^{2}}, & \text { se } & 5 \leq x<20 \\
1, & \text { se } x=20 \\
e^{-\frac{1}{2}\left(\frac{x-20}{10}\right)^{2}}, & \text { se } & 20<x \leq 50 \\
0, & \text { se } & x>50\end{array}\right.$ \\
\hline Média & $\mu_{T L}(x)=$ & $\left\{\begin{array}{lll}0, & \text { se } & x<20 \\
\frac{x-20}{30}, & \text { se } & 20 \leq x<50 \\
1, & \text { se } & x=50 \\
\frac{80-x}{30}, & \text { se } & 50<x \leq 80 \\
0, & \text { se } & x>80\end{array}\right.$ & $\mu_{T L}(x)=$ & $\begin{cases}0, & \text { se } x<20 \\
e^{-\frac{1}{2}\left(\frac{x-50}{10}\right)^{2}}, & \text { se } 20 \leq x \leq 80 \\
0, & \text { se } x>80\end{cases}$ \\
\hline Grande & $\mu_{T L}(x)=$ & 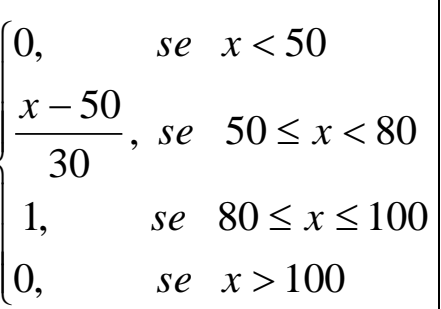 & $\mu_{T L}(x)=$ & $\left\{\begin{array}{lll}0, & \text { se } x<50 \\
e^{-\frac{1}{2}\left(\frac{x-80}{10}\right)^{2}} & \text { se } & 50 \leq x \leq 80 \\
1, & \text { se } & 80<x \leq 100 \\
0, & \text { se } & x>100\end{array}\right.$ \\
\hline
\end{tabular}

OBS: TL significa termo linguístico.

O Método da Média dos Máximos transforma a saída fuzzy em saída discreta encontrando a média aritmética ponderada entre os cores dos conjuntos fuzzy aos quais o elemento pertence, ponderadas pelo grau de pertinência, conforme a equação (2).

Já o Método do Centróide transforma a saída fuzzy em saída discreta encontrando a média aritmética entre os centros de gravidade dos conjuntos fuzzy aos quais o elemento pertence, ponderados pela área do conjunto, conforme a equação (3). 


$$
\begin{gathered}
y=\frac{\sum_{i=1}^{k} \mu_{i} \cdot y_{i}}{\sum_{i=1}^{k} \mu_{i}} \\
y=\frac{\sum_{i=1}^{k} a_{i} \cdot y_{i}^{\prime}}{\sum_{i=1}^{k} a_{i}}
\end{gathered}
$$

onde: $y$ é a saída discreta; $k$ é a quantidade de conjuntos fuzzy a que o elemento pertence; $\mu_{i}$ é o grau de pertinência com que o elemento pertence ao i-ésimo conjunto fuzzy; $y_{i}$ é o core do i-ésimo conjunto fuzzy a que o elemento pertence; $a_{i}$ é a área do i-ésimo conjunto fuzzy a que o elemento pertence; $y_{i}^{\prime}$ é o centro de gravidade do i-ésimo conjunto fuzzy a que o elemento pertence.

A análise envolve 84 dados. Depois de um estudo exploratório para avaliar algumas de suas propriedades técnicas (forma, tendência central, dispersão, lacunas e outliers), é utilizado um teste F com o objetivo de avaliar a influência nos resultados dos diferentes fatores considerados, bem como a possibilidade de interação. A análise é complementada com uma avaliação residual realizada com o objetivo de verificar algumas suposições da técnica utilizada a respeito de algumas propriedades dos dados. Finalizando, é calculado o coeficiente de Cronbach para identificar a combinação função de pertinência/método de "defuzzificação" que apresenta maior fidedignidade, o que é feito a partir dos resultados dos quatro fatores.

\section{RESULTADOS E DISCUSSÃO}

A Tabela 1 apresenta a média e o desvio padrão encontrados em cada uma das seis condições experimentais, bem como em cada nível de cada fator, mostrando a similaridade dos resultados tanto para a tendência central, como para a dispersão. Box plots (Figura 1) e gráficos de probabilidade normal não encontram indicativos de não normalidade muito acentuada.

O coeficiente de assimetria de Pearson, calculado para as 84 observações, indica assimetria bastante moderada e negativa ( $a_{\text {Pearson }}=-0,21$ ), enquanto que o coeficiente percentílico de curtose indica distribuição platicúrtica ( $k_{\text {percentiliko }}=0,30$ ), embora não muito acentuada.

Já o teste Levene não encontra evidências de heterogeneidade entre as variâncias $\left(f=0,11 ; g l_{1}=5 ; g l_{2}=78 ;\right.$ valorp $\left.=0,99\right)$. Esses achados possibilitam a realização da análise de variância por meio do teste $\mathrm{F}$, não sendo encontradas evidências de que os fatores função de pertinência e método de "defuzzificação" interfiram no resultado, conforme mostrado na Tabela 2. A análise gráfica dos resíduos não encontra indicativos de não normalidade acentuada, demonstrando a adequação da técnica utilizada.

A análise de confiabilidade realizada por meio do coeficiente de Cronbach (Tabela 3) indica que, na amostra investigada, os maiores resultados em cada função de pertinência são encontrados para as condições experimentais que consideram o método da média dos máximos para "defuzzificação". Neste método o resultado do coeficiente é maior quando a função de pertinência adotada é a triangular, razão pela qual a função triangular e o método da média dos máximos foram selecionados para serem utilizados nesses controladores fuzzy. 
Tabela 1 -Medidas Resumo

\begin{tabular}{|c|c|c|c|c|c|c|}
\hline \multirow{2}{*}{$\begin{array}{l}\text { Método de } \\
\text { "defuzzificação" }\end{array}$} & \multicolumn{2}{|c|}{ Função triangular } & \multicolumn{2}{|c|}{ Função gaussiana } & \multicolumn{2}{|c|}{ Total } \\
\hline & Média & $\begin{array}{l}\text { Desvio } \\
\text { padrão }\end{array}$ & Média & $\begin{array}{l}\text { Desvio } \\
\text { padrão }\end{array}$ & Média & $\begin{array}{l}\text { Desvio } \\
\text { padrão }\end{array}$ \\
\hline MC & 69,60 & 16,02 & 70,09 & 16,82 & 69,84 & 16,12 \\
\hline MM & 69,79 & 18,87 & 69,76 & 18,87 & 69,79 & 18,52 \\
\hline MMM & 71,87 & 18,17 & 71,79 & 18,43 & 71,83 & 17,96 \\
\hline Total & 70,42 & 17,32 & 70,55 & 17,64 & 70,49 & 17,37 \\
\hline
\end{tabular}

OBS: $\mathrm{MC}=$ Método do Centróide; $\mathrm{MM}=$ Método dos Máximos; MMM = Método da Média dos Máximos

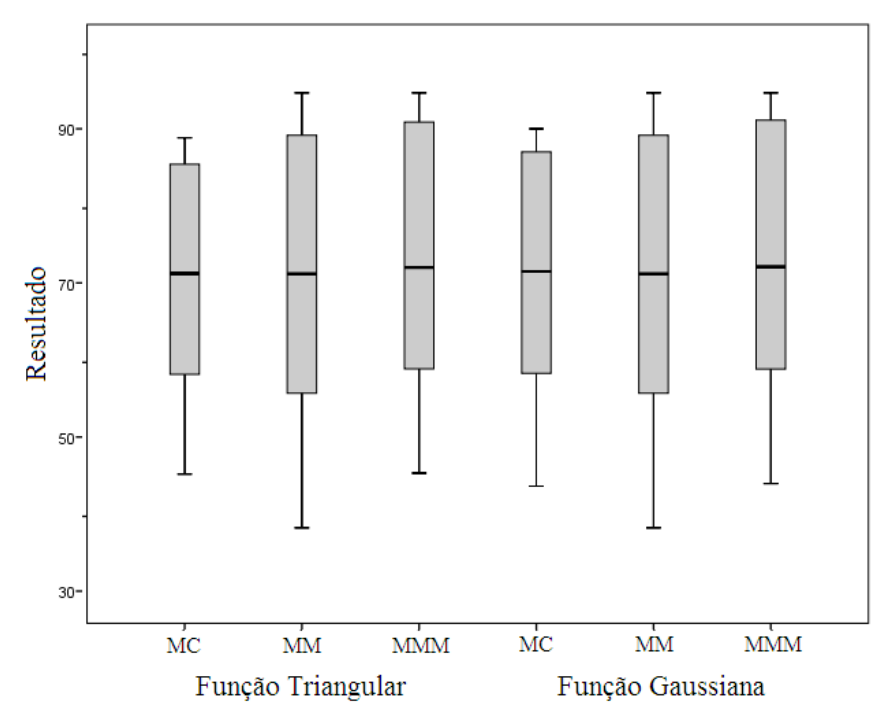

Figura 1 - Box Plots

Tabela 2 - Resultados da Análise de Variância

\begin{tabular}{lrrrrr}
\hline Fontes de variação & \multicolumn{1}{c}{$\boldsymbol{S}$} & $\boldsymbol{g} \boldsymbol{M}$ & \multicolumn{1}{c}{$\boldsymbol{M} \boldsymbol{F}$} & \multicolumn{1}{c}{$\boldsymbol{\text { valor } \boldsymbol { p }}$} \\
\hline Função de pertinência & 0,396 & 1 & 0,396 & 0,001 & 0,972 \\
Método de "defuzzificação" & 75,795 & 2 & 37,897 & 0,118 & 0,889 \\
Função * método & 1,356 & 2 & 0,678 & 0,002 & 0,998 \\
Erro & 442387,298 & 78 & 320,213 & & \\
\hline
\end{tabular}

Fonte: análise de dados.

Obs: $S Q=$ soma dos quadrados; $M Q=$ média dos quadrados; $g l=$ graus de liberdade; $F=$ estatística de teste; valor $p$ = probabilidade de ocorrência dos dados se não houvesse influência da variável/interação.

Tabela 3 - Resultados do Coeficiente de Cronbach

\begin{tabular}{lcc}
\hline "Defuzzificação" & Função Triangular & Função Gaussiana \\
\hline MC & 0,730 & 0,750 \\
MM & 0,685 & 0,685 \\
MMM & 0,759 & 0,755 \\
\hline
\end{tabular}

OBS: $\mathrm{MC}=$ Método do Centróide; $\mathrm{MM}=$ Método dos Máximos; $\mathrm{MMM}=$ Método da Média dos Máximos. 


\section{CONCLUSÃO}

$\mathrm{Na}$ literatura disponível existem vários estudos desenvolvidos empregando controladores fuzzy em diversos contextos. Entretanto, os critérios utilizados para a determinação da função de pertinência e do método de "defuzzificação" nem sempre são apresentados. Algumas vezes a escolha entre uma função de pertinência ou outra, entre um método de "defuzzificação" ou outro, é definida por especialistas que priorizam o conhecimento na área de aplicação.

Neste estudo, buscou-se avaliar por meio de técnicas estatísticas, a influência da utilização de dois tipos de função de pertinência e de três métodos de "defuzzificação" nos resultados da avaliação de um construto, feita por meio de controladores fuzzy. A primeira etapa da análise desenvolvida (análise de variância) não encontrou evidências de diferença significativa entre os resultados, sugerindo que a avaliação final do construto analisado parece não ser influenciada por essas propriedades dos controladores. No contexto analisado, tal definição foi auxiliada pelo coeficiente de Cronbach, o qual mede a fidedignidade da mensuração, sugerindo a utilização da função de pertinência triangular para "fuzzificação" e do método da média dos máximos para "defuzzificação".

Outros contextos, entretanto, necessitam ser estudados para avaliar melhor o efeito da função de pertinência e do método de "defuzzificação" usados em controladores fuzzy, o que se considera bastante pertinente. Melhorar a precisão de uma informação pode ser uma forma de melhorar a qualidade de uma tomada de decisão.

\section{AGRADECIMENTOS}

Os autores agradecem aos acadêmicos Leânia Moureira Agreda, Paulo Siga Thomaz, Gabriela Carré dos Santos, Franciele Muller Ribeiro, Deborah Cristiane Silva do Carmo e Jessica Fernandes Araújo pela colaboração na coleta de dados.

\section{REFERÊNCIAS BIBLIOGRÁFICAS}

1. Zadeh LA. The Concept of a Linguistic Variable and its Application to Approximate Reasoning-I. Information Science. 1975;8:199-249.

2. Malutta C. Método de apoio à tomada de decisão sobre adequação de aterros sanitários utilizando a Lógica Fuzzy [Tese]. Florianópolis (SC): Universidade Federal de Santa Catarina em Florianópolis; 2004. $221 \mathrm{p}$.

3. Simões M G, Franceschetti NN, Bose BK. Otimização de um sistema de geração de energia eólica através de controle fuzzy. SBA Controle \& Automação. 1999 jan-abr;10(1):48-58, doi: 10.1590/S0103-17592002000100001.

4. Souto KC. Sistema Especialista em Lógica Nebulosa para Cálculo em Tempo Real de Indicadores de Desempenho e Segurança na Monitoração de Usinas Nucleares [Tese]. Rio de Janeiro (RJ): Universidade Federal do Rio de Janeiro em Rio de Janeiro, 2005. 175 p.

5. Abadi DNM, Khooban MH. Design of optimal Mamdani-type fuzzy controller for nonholonomic wheeled mobile robots. Journal of King Saud University - Engineering Sciences. 2013 jan;27(1):1-9, doi: 10.1016/j.jksues.2013.05.003.

6. Sanaye S, Dehghandokht M, Fartaj A. Temperature control of a cabin in an automobile using thermal modeling and fuzzy controller. Applied Energy. 2013 mar;97(2):860-868, doi: 10.1016/j.apenergy.2012.02.078.

7. Mattos VLD, Pereira MC, Dimuro GP, Rosa SE. Proposta de modelo de avaliação da satisfação de usuários de transporte público usando lógica fuzzy. In: Quelhas OLG, Coordenador Geral. Proceedings of the VIII Congresso Nacional de Excelência em Gestão; 2012 jun 08-09; Rio de Janeiro, RJ. Rio de Janeiro (RJ): Firjan/UFF. c2012. p.1-14.

8. Viticoski RL, Agreda LM, Mattos VLD, O uso de controladores fuzzy na reconstrução de construtos: um estudo exploratório. In: Másculo, FS, Diretor Científico ABEPRO. Proceedings of XXXIII Encontro Nacional de Engenharia de Produção, 2013 out 13-16; Salvador, BA. Rio de Janeiro (RJ), Abepro, c2013. p.1-10. 